\title{
Electron beam optics and trajectory control in the Fermi free electron laser delivery system
}

\author{
S. Di Mitri, M. Cornacchia, and C. Scafuri \\ Sincrotrone Trieste, Basovizza (TS), Italy \\ M. Sjöström \\ MAX-lab, Lund, Sweden \\ (Received 18 May 2011; published 19 January 2012)
}

\begin{abstract}
Electron beam optics (particle betatron motion) and trajectory (centroid secular motion) in the FERMI@Elettra free electron laser (FEL) are modeled and experimentally controlled by means of the ELEGANT particle tracking code. This powerful tool, well known to the accelerator community, is here for the first time fully integrated into the Tango-server based high level software of an FEL facility, thus ensuring optimal charge transport efficiency and superposition of the beam Twiss parameters to the design optics. The software environment, the experimental results collected during the commissioning of FERMI@Elettra, and the comparison with the model are described. As a result, a matching of the beam optics to the design values is accomplished and quantified in terms of the betatron mismatch parameter with relative accuracy down to the $10^{-3}$ level. The beam optics control allows accurate energy spread measurements with sub-keV accuracy in dedicated dispersive lines. Trajectory correction and feedback is achieved to a $5 \mu \mathrm{m}$ level with the implementation of theoretical response matrices. In place of the empirical ones, they speed up the process of trajectory control when the machine optics is changed, avoid particle losses that may occur during the on-line computation of experimental matrices, and confirm a good agreement of the experimental magnetic lattice with the model.
\end{abstract}

DOI: 10.1103/PhysRevSTAB.15.012802

PACS numbers: 29.20.Ej, 41.85.-p, 07.07.Tw

\section{INTRODUCTION}

The FERMI@Elettra single-pass, linac-based free electron laser (FEL) at the Elettra Laboratory of Sincrotrone Trieste [1] is one of the FEL based European projects. Currently in the commissioning stage, it purports to become the international user facility in Italy, providing high brilliance x-ray pulses for scientific investigations of very fast and high resolution processes in material science and physical biosciences. FERMI is a seeded FEL optimized for high gain harmonic generation [2-5]. This scheme makes the FEL performance particularly sensitive to the electron beam optics and energy distribution. Thus, control of the particle transverse motion and characterization of the energy distribution are of critical importance for the machine operation.

In its first part, this article focuses on the ELEGANT-based [6] on-line control of the electron beam optics. In spite of many existing works on this topic [7-10], this article first shows the full integration of ELEGANT into the Tangoserver [11] based high level software of an FEL facility, thus ensuring optimal charge transport efficiency and successful transfer of the beam Twiss parameters to the design

Published by the American Physical Society under the terms of the Creative Commons Attribution 3.0 License. Further distribution of this work must maintain attribution to the author $(s)$ and the published article's title, journal citation, and DOI. optics. The betatron mismatch parameter [12] has been measured with an error as small as $10^{-3}$.

We also show that such accurate beam optics control allows very precise energy spread measurement in dedicated dispersive lines. In comparison with demonstrated performance as reported in [13-16], our sub-keV accuracy is quite good.

We finally report about the ELEGANT-based trajectory manipulation along the FERMI beam delivery system. A trajectory control to a $5 \mu \mathrm{m}$ level is achieved with the implementation of theoretical response matrices. The adoption of theoretical matrices for the entire machine, versus standard methods that are based on the measurement or postprocessing of empirical response matrices [17-20], speeds up the process when sizable changes are made to the lattice, avoids particle losses that may occur during the computation of experimental matrices, and confirms the agreement of the experimental magnetic focusing with the model.

The article is organized as follows. After an introduction to the FERMI layout given in Sec. II, Sec. III shows how the ELEGANT code has been interfaced with the real machine. Section IV describes the procedure and the experimental results of the beam optics matching and transport. The betatron mismatch parameter is measured to evaluate the validity of the matching algorithm. According to the model, the optics matching algorithm optimizes the resolution of the energy spread measurement, which is then used to indirectly verify the control of the Twiss 
parameters in the FERMI spectrometer lines, as discussed in Sec. V. In Sec. IV, the use of ELEGANT-generated theoretical trajectory response matrices (TRM) is described. The theoretical matrices are uploaded into a feedback tool, which is also used for trajectory correction. The successful operation of the trajectory correction loop with such theoretical matrices, even when applied to large portions of the accelerator, confirms that the machine optics is well described by the model.

\section{FERMI LAYOUT}

FERMI is the new normal conducting, $3 \mathrm{GHz}$ linacdriven seeded FEL currently being commissioned in Trieste, Italy. The linac is providing a $1.2 \mathrm{GeV}$ electron beam which drives two seeded FELs, called FEL1 and FEL2, in the wavelength range of $100-4 \mathrm{~nm}$. Wavelength tunability and variable photon polarization will also be provided to the users with the variable gap APPLE-II type undulators [21]. Table I summarizes the main facility parameters and performance. A description of the project and of the most recent commissioning results is given in [22-26]. Figure 1 shows a conceptual layout of the FERMI linac and undulators. The present lattice includes: 92 quadrupole magnets with integrated gradient in the range 0.4-7.7 $\mathrm{T}$; 62 steering magnets per plane, providing a maximum kick angle in the range
1.0-1.5 mrad; 48 strip-line beam position monitors (BPMs) and $14 \mathrm{rf}$ cavity beam position monitors (CBPMs). These are installed in front of and along the undulator line. The single shot resolution of the BPMs is estimated to be $5 \mu \mathrm{m}$ for a bunch charge bigger than 50 pC. The CBPMs are complex systems, developed in house at the Elettra laboratory, and are being commissioned [27]. Their present resolution is $2 \mu \mathrm{m}$ at a bunch charge higher than $50 \mathrm{pC}$. Several screen systems that mount yttrium aluminum garnet and optical transition radiation targets are used to measure the electron beam transverse size. The main linac layout includes three dispersive lines for destructive beam diagnostics. They are called SPLH, SPBC1, and DBD. They are located, respectively, at the end of the laser heater (LH) area, at the beam energy of $100 \mathrm{MeV}$; at the end of the first magnetic compressor (BC1) diagnostic area, at the beam energy of $350 \mathrm{MeV}$; and at the end of the FERMI linac, at the beam energy of $1.2 \mathrm{GeV}$. They are sketched in Fig. 1. In each of these lines, the mean energy of the beam is measured by centering the beam spot on a screen, previously aligned with a laser tracker [28] with an accuracy of $300 \mu \mathrm{m}$, and computing the beam energy from the dipole magnet current-to-energy calibration table. The dipole magnets calibration table has been experimentally built by the manufacturer with a relative error equal or smaller than $10^{-4}$.

TABLE I. FERMI@Elettra main parameters and performance.

\begin{tabular}{|c|c|c|c|c|}
\hline & FEL1 commissioning & FEL1 operation & FEL2 operation & Units \\
\hline \multicolumn{5}{|l|}{ Machine parameters } \\
\hline Linac frequency & & 2.998 & & $\mathrm{GHz}$ \\
\hline BC1 energy & & 350 & & $\mathrm{MeV}$ \\
\hline $\mathrm{BC} 1 R_{56}$ & -49 & -41 & -41 & $\mathrm{~mm}$ \\
\hline BC2 energy & & 670 & & $\mathrm{MeV}$ \\
\hline $\mathrm{BC} 2 R_{56}$ & 0 & -30 & -30 & $\mathrm{~mm}$ \\
\hline Total compression factor & 6 & 11 & 11 & \\
\hline FEL scheme & HGHG 1-stage & HGHG 1-stage & HGHG 2-stage & \\
\hline Total length & & 310 & & $\mathrm{~m}$ \\
\hline \multicolumn{5}{|l|}{ Electron beam parameters } \\
\hline Energy & 1.2 & $0.9-1.2$ & $1.2-1.5$ & $\mathrm{GeV}$ \\
\hline Charge & 0.35 & 0.8 & 0.8 & $\mathrm{nC}$ \\
\hline Slice norm. emittance (rms) & 1.0 & 1.0 & 1.0 & $\mathrm{~mm} \operatorname{mrad}$ \\
\hline Slice energy spread (rms) & 0.1 & 0.2 & 0.2 & $\mathrm{MeV}$ \\
\hline Peak current (flat region) & $<200$ & 500 & 800 & A \\
\hline Bunch duration (FWHM) & 1.0 & 0.7 & 0.7 & ps \\
\hline \multicolumn{5}{|l|}{ Photon beam parameters } \\
\hline Output wavelength range & $40-60$ & $20-100$ & $4-20$ & $\mathrm{~nm}$ \\
\hline Output pulse duration (rms) & 150 & 50 & 50 & fs \\
\hline Bandwidth (rms) & $20-40$ & 17 (at $40 \mathrm{~nm})$ & $5($ at $10 \mathrm{~nm})$ & $\mathrm{meV}$ \\
\hline Polarization & Variable & Variable & Variable & \\
\hline Peak power & 0.1 & $1-5$ & $0.3-1$ & GW \\
\hline Photons per pulse & $10^{12}$ & $10^{13}$ & $10^{12}$ & in $1 \mathrm{meV}$ bw \\
\hline Repetition rate & 10 & 50 & 50 & $\mathrm{~Hz}$ \\
\hline
\end{tabular}




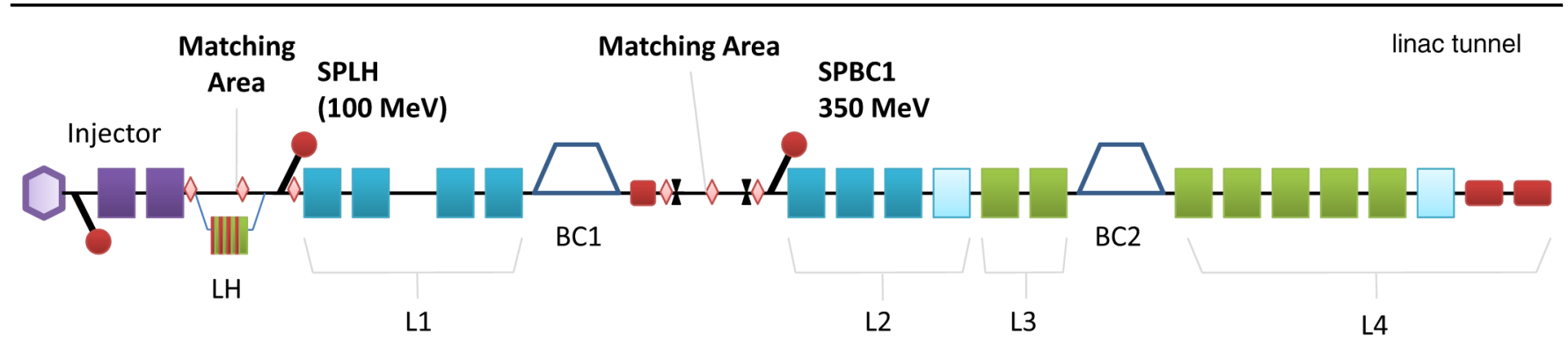

FEL1: 1 modulator +1 dispersive section +6 radiators

undulator hall

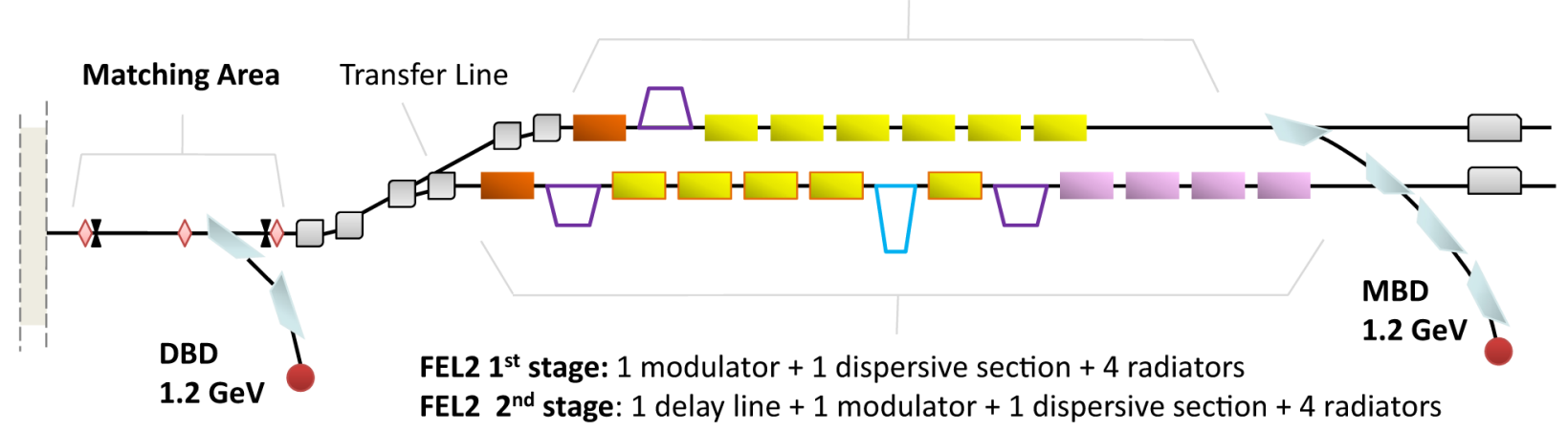

FIG. 1. Split layout of the FERMI FEL (conceptual, not to scale). The linac includes the injector, the laser heater (LH) area, different types of accelerating structures_-represented with different colors-accommodated in linac 1 (L1), linac 2 (L2), linac 3 (L3), and linac 4 (L4), two magnetic chicanes for bunch length compression (BC1 and BC2), three optics matching area, the laser heater spectrometer line (SPLH), the first compressor spectrometer line (SPBC1), and the diagnostic beam dump (DBD). A transfer line brings the electron beam to the FEL1 or FEL2 undulator line (different undulator segments are represented by different colors), followed by the main beam dump (MBD) line.

\section{ELEGANT ON-LINE}

The ELEGANT code has extensively been used during the design of the FERMI electron beam delivery system [1,22]. We have decided to use it also for performing the optics matching and providing the optics model for trajectory control on the live machine. In order to use it as an online machine model, we have developed a set of utilities for interfacing the simulator with the accelerator. We have exploited the ELEGANT capability to read and write a set of element parameters from a file in self-describing data sets (SDDS) format [6] via the LOAD_PARAMETERS and WRITE_PARAMETERS commands. The SDDS file is filled in with the actual parameters of the running accelerator by means of a dedicated utility which examines the SDDS files and maps the requested parameters to the appropriate control system variables by means of database tables. The requested variables are then acquired from the control system and scaled if necessary (e.g. converting BPM readings from millimeter to meter). The reverse path is followed for setting parameters: ELEGANT writes the new values to the SDDS files, another dedicated utility reads the new parameters from the SDDS file, maps them to control variables, and sets the values via standard control system calls.
Since ELEGANT works with normalized machine physics quantities, we have developed a set of specialized control system servers, called Tango devices [29], which perform the conversion from engineering quantities (e.g. current) to machine physics quantities (e.g., quadrupole strength) by means of calibration tables. Such tables are directly handled by the Tango server [11]. As an example, the interface of the machine with ELEGANT requires the determination of the quadrupole strength, $k$ in $\mathrm{m}^{-2}$, and the steering magnets angular kick, $\theta$ in mrad, as a function of the supplied current, in A. Well-known relations as in [30] are used for the conversions. The Tango devices are also directly accessible to operators by means of graphical control panels.

\section{OPTICS CONTROL}

The goal of optics matching is to impose the design values of the Twiss functions to the electron beam. Groups of (at least) four quadrupole magnets are installed for optics matching at several locations of the FERMI beam line. During commissioning, matching is routinely carried out at three locations: at the entrance of the $\mathrm{LH}$ area, at the exit of $\mathrm{BC} 1$, and at the linac end (see Fig. 1). 
From the cathode up to the end of the injector, where the beam energy is $100 \mathrm{MeV}$, the beam optics cannot be predicted with sufficient accuracy in ELEGANT since the particles move in the space-charge dominated regime. For this reason, it is very important to measure the beam optics at the end of the injector, where the electron spatial distribution is frozen to any practical purpose. To improve the optics control, the measurement of the Twiss parameters and the matching procedure is usually repeated in the $\mathrm{BC} 1$ and linac end region. In all cases, the beam Twiss parameters are measured by using the method of quadrupole scan [31,32].

A MATLAB [33] script reads the present quadrupole setup and transfers this information to ELEGANT. Starting from the measured Twiss parameters, the code back-propagates the optical functions with a reverse ordered lattice file up to a conventional point upstream of the matching station. The initial conditions of the Twiss parameters for the present beam optics are thus available at the entrance of the matching station. ELEGANT is then run in the common forward-tracking mode in order to match the beam to the desired nominal lattice and to evaluate the optical functions along the downstream part of the accelerator. This loop is illustrated in Fig. 2. All information about the matching loop result is accessible to the user. The matching loop has been coded in MATLAB and a MATLAB graphical user interface is available as a standard control room application. The matching procedure is quite reliable,
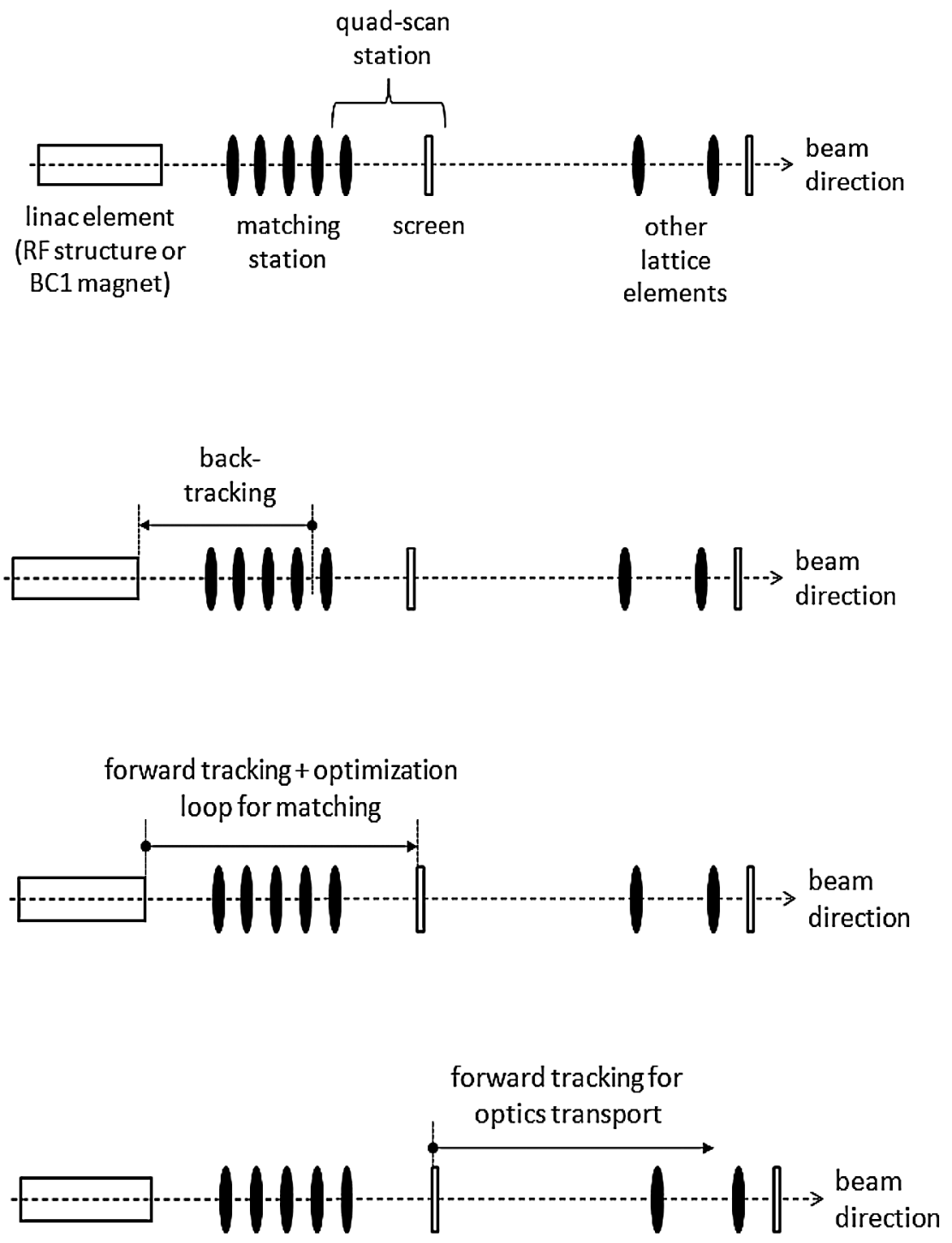

FIG. 2. Illustration of the optics matching loop. From top to bottom: (i) the beam Twiss parameters are measured with the quadrupole scan technique at the entrance of the last quadrupole magnet of the matching station; (ii) the present machine configuration is read by ELEGANT and the measured Twiss parameters are back-tracked to a point upstream of the matching station; (iii) starting from the present machine configuration, ELEGANT starts optimizing the quadrupole strengths to match the beam Twiss parameters to the design values; (iv) once the matching has been performed, the beam is transported through the downstream lattice. 


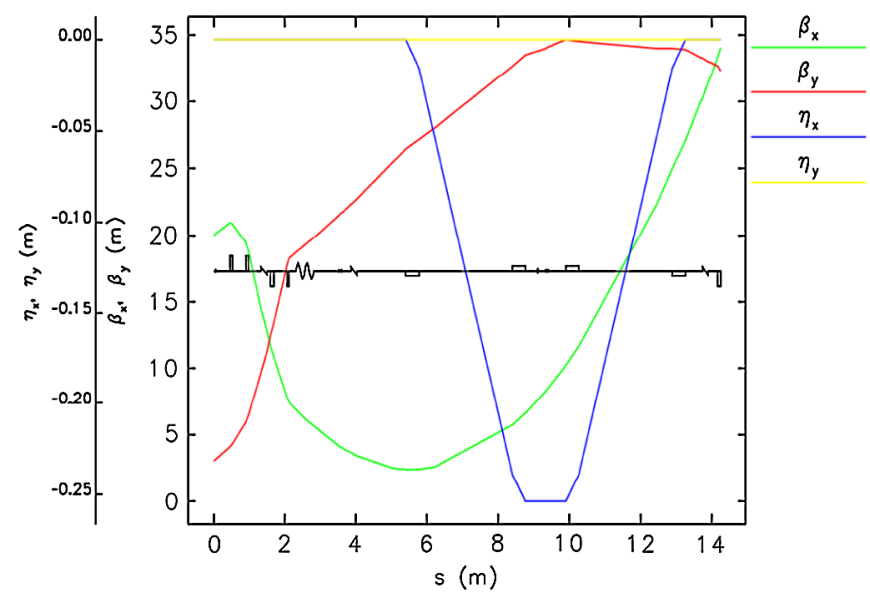

FIG. 3. The optical functions, measured with quadrupole scan in the $\mathrm{BC} 1$ area, are back-propagated in the lattice-from the end of the $\mathrm{BC} 1$ matching station (left side of the plot) to the entrance of the $\mathrm{BC} 1$ magnetic chicane (right side of the plot) — on the basis of the quadrupole strengths read by ELEGANT. The Twiss parameters at the entrance of the matching station are thus available to start the matching loop. The result of the matching loop is shown in Fig. 4. The chicane bending angle is set to $85 \mathrm{mrad}$. The black line in the middle sketches the machine layout.

usually converging in a couple of iterations. One can compare the matching result with the design Twiss parameters before actually setting the calculated quadrupole strengths. One can also improve the matching result by iterating the procedure. A theoretical betatron mismatch parameter is defined as follows [12]:

$$
\xi=\frac{1}{2}(\bar{\beta} \gamma-2 \bar{\alpha} \alpha+\bar{\gamma} \beta)
$$

where $\bar{\beta}, \bar{\alpha}, \bar{\gamma}$ are the design Twiss parameters, for each transverse plane. $\beta, \alpha, \gamma$ are the Twiss parameters computed by ELEGANT at the end of the optimization process. By definition, $\xi \geq 1$; the closer this value is to 1 , the closer the ELEGANT solution is to the design optics. A graphical output is displayed that shows the betatron functions as they are computed in the back-tracking mode (prior to matching, see Fig. 3) and in the forward-tracking mode (after the optics matching has been computed, see Fig. 4). All intermediate data and results of the matching procedure are exchanged via SDDS format files and can be plotted with standard SDDS based tools.

The ELEGANT model of the electron optics includes the vertical edge field focusing of the $\mathrm{BC} 1$ dipole magnets as well as the rf edge focusing of the accelerating structures, which depends in turn on the accelerating gradient and the beam energy. Space-charge forces are not taken into account because, as mentioned above, they do not play an important role downstream of the injector (see also [22,23] for the study of space-charge forces in the $\mathrm{LH}$ and the $\mathrm{BC} 1$ area). At the same time, second order transport matrices are implemented in ELEGANT. The machine model and matching procedure therefore include high order effects such as

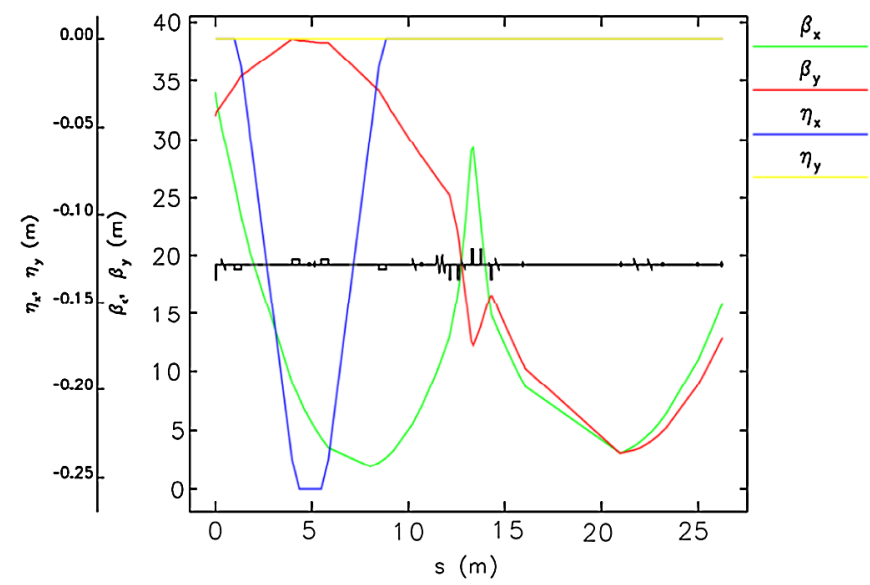

FIG. 4. Some quadrupoles downstream of BC1 are used in the ELEGANT matching procedure to match the beam optics to the design values. In this plot, the optical functions are transported in the common forward mode from the end point of Fig. 3 (entrance of $\mathrm{BC} 1$ ) to the end of the $\mathrm{BC} 1$ diagnostic area. The matching loop performed by ELEGANT finds a good solution after one iteration (a low- $\beta$ symmetric optics after $\mathrm{BC} 1$ reflects the design optics). The theoretical mismatch parameters in this case are $\xi_{x}=1.002, \xi_{y}=1.005$. The black line in the middle sketches the machine layout.

geometric and chromatic aberrations. However, the design optics has been built in a way that such perturbations are not expected to be important [1].

Figure 3 shows, as an example, the betatron functions in the $\mathrm{BC} 1$ area computed by ELEGANT in the back-tracking mode during one commissioning shift. Figure 4 shows the betatron functions computed in the forward-tracking mode. These correspond to the solution found by the ELEGANT matching loop for this area. The quadrupole strengths foreseen by this solution are uploaded to the machine. In order to confirm the success of the ELEGANT computation and the real matching of the electron beam optics to the design lattice, the beam Twiss parameters are measured again after matching and compared with the theoretical expectation in order to evaluate the experimental betatron mismatch parameter. This has actually been measured in several occasions during the FERMI commissioning and under different machine and beam conditions. Some measurement results are shown in Figs. 5 and 6. The central value of the experimental mismatch parameter is usually very close to the target value of 1 in both planes. The measurement error on the values of the Twiss parameters yields a maximum error of the mismatch parameter as shown by the error bars. In many cases, this error reaches the small value of $10^{-3}$ (see [34] for the state-of-art beam optics measurement and matching in a FEL facility). The measurement error of the Twiss parameters is evaluated on the basis of the fitting procedure that is applied to the result of the quadrupole scan and on the uncertainty brought by the measurement of the beam size. This is affected both from the $30 \mu \mathrm{m}$ resolution of the screen system and from 


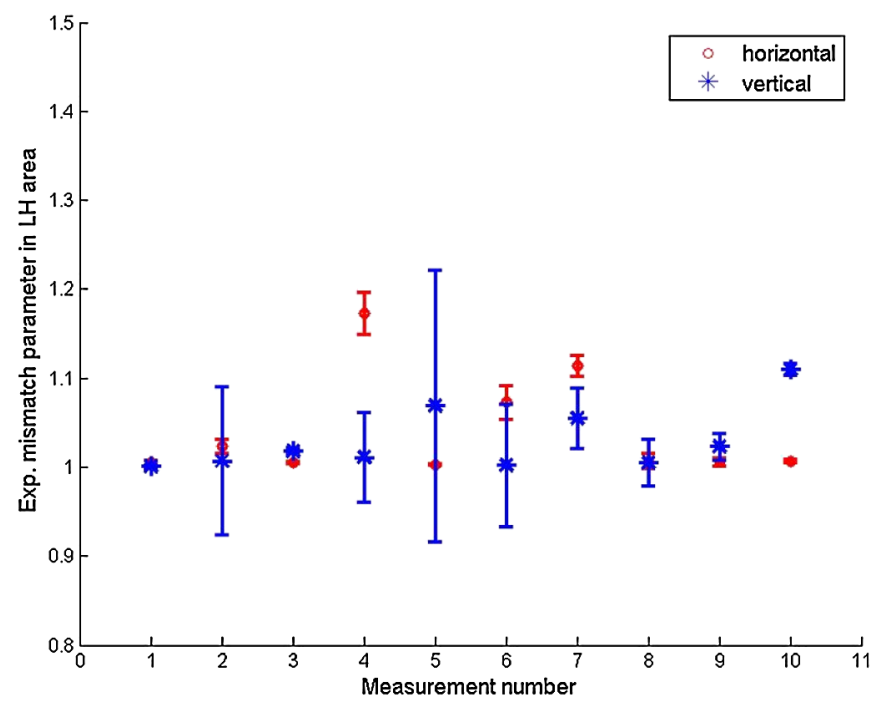

FIG. 5. Betatron mismatch parameter measured at one location in the LH area vs measurement number. Each value is the result of a single measurement of the Twiss parameters. The mismatch parameter is measured after the matching loop has been performed with ELEGANT and the quadrupole strengths applied in the LH area. (Notice that central values smaller than 1 have no physical sense: the full range of the vertical axis is used to show the full error bars associated with the measurements.)

the $\sim 10 \mu \mathrm{m}$ shot-to-shot variation of the rms beam size (the quadrupole scan is usually performed over many shots, at the repetition rate of $10 \mathrm{~Hz}$ ). We have noticed that the quality of the beam optics matching process deteriorates

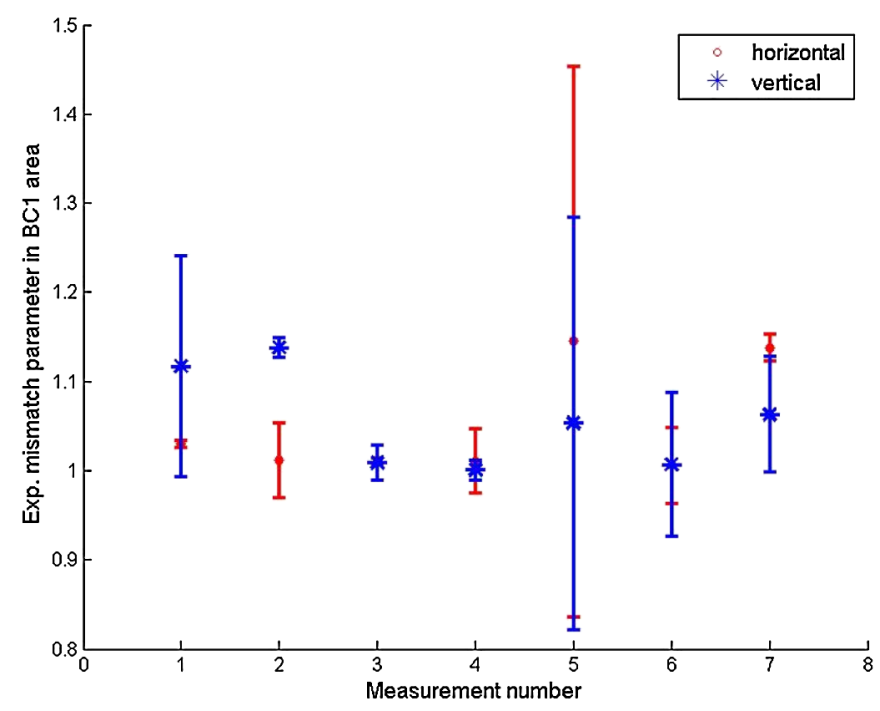

FIG. 6. Betatron mismatch parameter measured at one location in the $\mathrm{BC} 1$ area vs measurement number. Each value is the result of a single measurement of the Twiss parameters. The mismatch parameter is measured after the matching loop has been performed with ELEGANT and the quadrupole strengths applied in the $\mathrm{BC} 1$ area. (Notice that central values smaller than 1 have not physical sense: the full range of the vertical axis is used to show the full error bars associated with the measurements). when the measured beam presents strong asymmetries between the horizontal and the vertical plane or in the presence of beam size jitter much bigger than the aforementioned value. In these cases, the betatron mismatch parameter has very large errors (this is the case for the largest error bars in Figs. 5 and 6).

\section{ENERGY SPREAD MEASUREMENT}

The FERMI linac includes three spectrometer lines, SPLH, SPBC1, and DBD (see Fig. 1), in which the beam energy distribution is characterized by analyzing the transverse profile collected on a screen, in the bending plane. The minimum relative energy deviation $\delta=\left(E_{0}-E_{1}\right) / E_{0}$ between two particles with energy $E_{0}$ and $E_{1}$ corresponds to the physical separation $\Delta$ of the two particle projections onto the screen:

$$
\delta=2 \Delta / \eta
$$

where $\eta$ is the dispersion function in the bending plane at the screen location. By definition, Eq. (2) shows the intrinsic resolution of the energy deviation measurement. We now consider the contribution of the betatron particle motion to the chromatic beam size, in the assumption of a Gaussian particle distribution:

$$
\sigma=\sqrt{\varepsilon \beta+\left(\eta \sigma_{\delta}\right)^{2}}=\eta \sigma_{\delta} \sqrt{1+\kappa^{2}},
$$

where we have defined the coefficient $\kappa=\sqrt{\varepsilon \beta} /\left(\eta \sigma_{\delta}\right)$. Given the pure chromatic beam size $\sigma_{0}=\eta \sigma_{\delta}$, the relative error of the energy spread measurement induced by the particle betatron motion is

$$
\frac{\sigma-\sigma_{0}}{\sigma_{0}}=\sqrt{1+\kappa^{2}}-1
$$

Figure 7 plots Eq. (4) for the design optics in SPBC1, as a function of the energy spread central value. In this specific case, the rms energy spread is expected to be in the range $0.05 \%-1.00 \%$ for a mean energy of the electron beam in the range $100-350 \mathrm{MeV}$. The relation $\sigma_{\delta} \cong \sigma / \eta$ is valid only when $\kappa \ll 1$ or, in other words, when the spectrometer resolving power $\eta / \sqrt{\beta} \gg 1 \mathrm{~m}^{1 / 2}$. This condition sets important constraints on the beam line design and, at the same time, it requires the electron beam be perfectly matched to the design optics of the lattice. So, since the result of the energy spread measurement depends on the betatron and dispersion function at the screen location, we expect a smaller energy spread value and a measurement accuracy approaching the theoretical resolution of the spectrometer line when the beam is matched.

The resolution of the screen system, due to the target granularity, the optical path of the collected light, and the resolution of the CCD camera, is $30 \mu \mathrm{m}$. Although the beam size is much larger than this $(\sigma \geq 1 \mathrm{~mm})$, a detailed 
TABLE II. Design optical parameters of the three spectrometer lines in the FERMI main linac. For comparison, the values in parentheses refer to the optics used for FEL production, not for the energy spread measurement.

\begin{tabular}{lccc}
\hline \hline & SPLH & SPBC1 & DBD \\
\hline$\beta_{x}$ at the screen [m] & $2.9(19.0)$ & $0.2(10.4)$ & $2.0(4.9)$ \\
$\alpha_{x}$ at the screen & $-0.7(-2.5)$ & $<0.1(-1.5)$ & $1.4(-1.0)$ \\
$\left|\eta_{x}\right|$ at the screen [m] & 1.7 & $0.6(1.0)$ & $1.2(1.8)$ \\
$\eta_{x}$ at the screen [rad] & -0.8 & $<-0.1(-0.4)$ & $-0.6(0.6)$ \\
$\eta_{x} / \sqrt{\beta_{x}}$ at the & $1.0(0.4)$ & $1.3(0.3)$ & $0.8(0.8)$ \\
screen $\left[\mathrm{m}^{1 / 2}\right]$ & & & \\
\hline \hline
\end{tabular}

analysis of the error propagation shows that when the resolving power of the spectrometer line is maximized and the beam is well matched to the design optics, the contribution of the screen resolution starts dominating the energy spread measurement error. In this case, we have to evaluate the measurement error in a more formal way than in Eq. (4). We compute the energy spread by subtracting the geometric beam size to the measured one, $\sigma_{\delta}=$ $\sqrt{\sigma^{2}-\varepsilon \beta} / \eta$ and then we compute the error propagation of this expression. The complete analytical treatment is given in the Appendix.

Table II lists the optical parameters of the three spectrometer lines in the FERMI layout. Table III shows the energy spread predicted by ELEGANT, measured before and after the ELEGANT-based optics control. The ELEGANT simulation has been carried out with a $350 \mathrm{pC}, 6 \mathrm{ps}$ (FWHM) long beam, accelerated on-crest along the entire linac. The error on the energy spread is evaluated with the formalism described in the Appendix. Initially (before optics matching) we have assumed some optics uncertainty in the spectrometer region (for a badly matched beam such as $\xi \gg 1$, we expect $\beta_{x}$ as large as 50 times, and $\eta_{x}$ smaller by a factor 2 than the design value). After matching, the energy spread value diminishes and approaches the simulated result. A measured value for the energy spread smaller than in the simulation, for all three spectrometer lines, suggests that the real bunch length is shorter than 6 ps. The optics performance of the spectrometer lines allows us to reach sub-keV accuracy for the energy spread measurement in SPLH; the "worse" case of $6 \mathrm{keV}$ accuracy is obtained at $1.2 \mathrm{GeV}$. This is a promising result, especially in the view of the possibility of measuring the slice energy spread at the same locations.

\section{TRAJECTORY CONTROL}

The FERMI trajectory feedback tool has some novel capabilities to perform trajectory control with respect to standard methods, which are normally adopted both in rings and linacs and are based on the measurement or postprocessing of empirical response matrices. The first point of our work is that a theoretical TRM (direct or inverse) computed by ELEGANT can be imported, possibly compared with the one measured via the visualization tool and used for trajectory correction. The adoption of theoretical matrices for the entire machine versus empirical ones has two advantages. First, it speeds up the process of trajectory control when some large changes are applied to the quadrupole magnet strengths. In this case, a new TRM can immediately be computed, uploaded, and applied to the trajectory control without affecting the facility operation. Second, it avoids particle losses that may occur during the measurement of matrices along the $300 \mathrm{~m}$ long beam delivery system. The successful correction with theoretical TRMs confirms the agreement of the effective magnetic focusing with the model. The TRM, either theoretical or experimental, can be visualized in a 3D contour plot to identify areas of particularly high or poor BPMs sensitivity to the corrector strengths.

The second important point of our work is that experimental and theoretical TRMs can be merged for global trajectory manipulation. This typically happens when a TRM is measured in the injector or in the undulator area and then merged with the theoretical TRM for the main linac. As previously mentioned, the ELEGANT machine model does not include the space-charge dominated area of the FERMI injector. Also the undulator area is not well modeled yet in the presence of the APPLE-II type undulators [21]. These devices may have a large impact on the beam focusing, depending on their gap and phase setup. Thus, a global matrix is routinely used for trajectory control from the gun to the FEL area, which is the merged version of two experimental and one theoretical TRM.

During trajectory correction, the position of the beam centroid is usually forced onto the design trajectory, which should coincide with the zero reading of the BPMs, barring any calibration errors. However, other trajectories are sometimes desirable. For example, we use trajectory bumps to maximize the transmission efficiency in the presence of residual magnetic field across several

TABLE III. Mean energy and rms energy spread, simulated and measured at three FERMI spectrometer lines, respectively before and after the ELEGANT-based optics control.

\begin{tabular}{lccccc}
\hline \hline & $\begin{array}{c}\text { Mean energy predicted } \\
\text { by ELEGANT [MeV] }\end{array}$ & $\begin{array}{c}\sigma_{E} \text { predicted by } \\
\text { ELEGANT [keV] }\end{array}$ & $\begin{array}{c}\text { Mean energy } \\
\text { measured [MeV] }\end{array}$ & $\begin{array}{c}\sigma_{E} \text { measured before } \\
\text { optics matching [keV] }\end{array}$ & $\begin{array}{c}\sigma_{E} \text { measured after } \\
\text { optics matching [keV] }\end{array}$ \\
\hline SPLH & 99.8 & 73 & $97.95 \pm 0.06$ & $84 \pm 20$ & $66.7 \pm 0.4$ \\
SPBC1 & 350.2 & 280 & $347.5 \pm 0.2$ & $341 \pm 170$ & $180.2 \pm 3.5$ \\
DBD & 1206.4 & 522 & $1210.2 \pm 0.8$ & $611 \pm 200$ & $465.2 \pm 6.0$ \\
\hline \hline
\end{tabular}




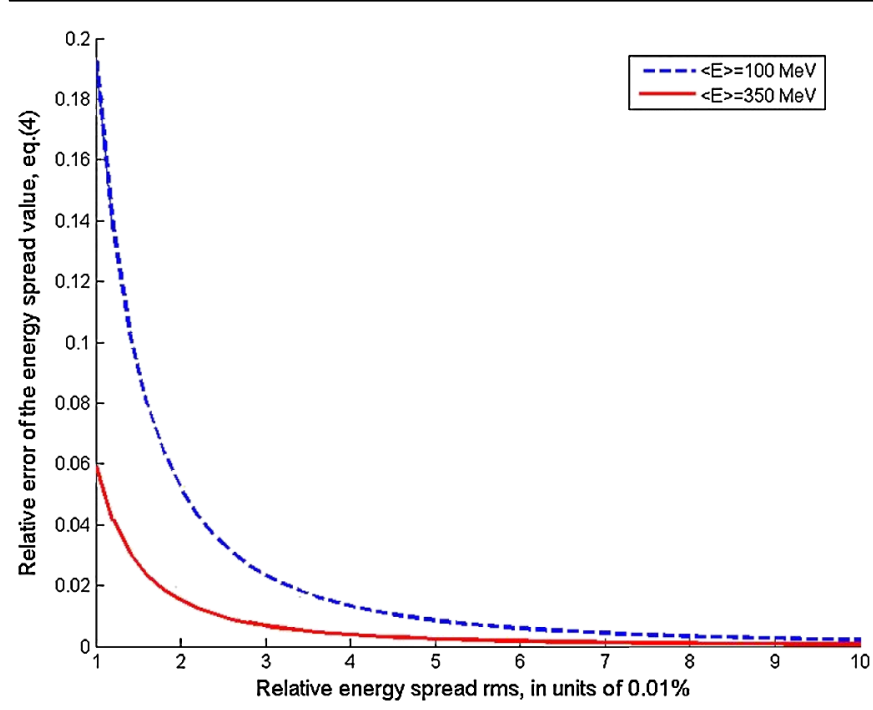

FIG. 7. Relative error of the relative energy spread vs its central value, as depicted in Eq. (4), for the SPBC1 line. The dotted line is for the beam mean energy of $100 \mathrm{MeV}$, the solid line is for $350 \mathrm{MeV}$.

spectrometer magnets of the FERMI beam line. We also adopt some bumps to compensate the short-range transverse wake field instability [35]. Moreover, there are uncertainties associated with the electronic center or the mechanical alignment of the BPMs. If the BPM offsets are not known a priori and possibly larger than the alignment specification or if some trajectory bumps are required, then a good strategy is to reduce the rms strength of the correctors and to pay less attention to the absolute beam position. The technique based on the singular value decomposition (SVD) [36] allows the minimization of the corrector rms strength while correcting the trajectory.

The SVD formalism is implemented in the MATLABbased trajectory feedback for FERMI that is routinely used both for trajectory control and feedback operation at approximately $0.7 \mathrm{~Hz}$. In particular, the program is capable of displaying any response matrix, as well as its inversion using either regular SVD with all singular values [36], truncated singular value decomposition (TSVD) [37] or SVD with Tikhonov regularization [38]. It is also possible to view the singular values and how they have been modified for all three inversion options. For the TSVD, the given tolerance specifies how small singular values will be included in the inversion. In case the matrix rank is lower than both the number of actuators or sensors, some singular values should be removed in order for the inversion to produce a matrix with noninfinite elements. As for the Tikhonov inversion option, small singular values are scaled up, which allows inversion of low rank matrices. In more detail, given the singular value decomposition of $A$ with singular values $w_{i}$, the Tikhonov regularized solution can be expressed as [38]
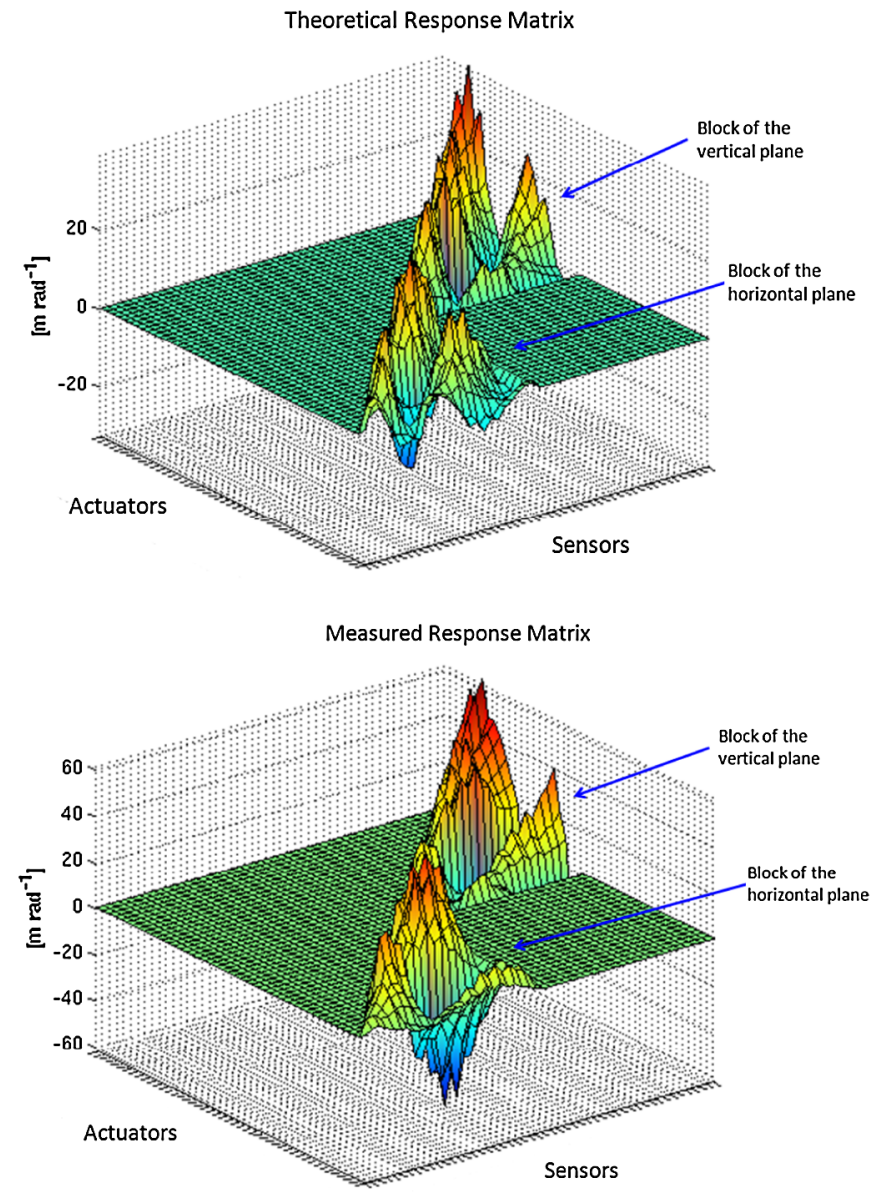

FIG. 8. Visualization of direct TRMs. Theoretical (top) and measured (bottom) TRM for the FERMI linac. The two diagonal blocks are for the horizontal and vertical plane, respectively. The "theoretical" TRM shown on the top is the merged version of a two-dimensional experimental TRM for the injector and of the theoretical TRM for the main linac. The merged matrix has been used for global trajectory correction in the FERMI linac, as shown in Figs. 9 and 10.

$$
\bar{\theta}=V D U^{t} \Delta x,
$$

where $D$ has diagonal values $D_{i i}=w_{i} /\left(w_{i}^{2}+q^{2}\right)$ and is zero elsewhere. The singular value scaling means the solution obtained minimizes the following norm:

$$
\|A \theta-\Delta x\|^{2}+\|\Gamma \theta\|^{2}
$$

for a Tikhonov matrix $\Gamma=q \cdot I$, where $q=1, \ldots, n$ is a positive integer. Equation (6) then shows the utility of the scaling parameter $q$ : it specifies the importance of minimizing the corrector strengths relative to minimizing the trajectory deviations. A value of zero will mean no weight is given to the amplitude of corrector changes when the solution is computed, while an increasing value will increase the weight on minimizing the norm of corrector changes.

The tool is also used to launch parallel feedback loops on the beam line. The possible interference of one loop 

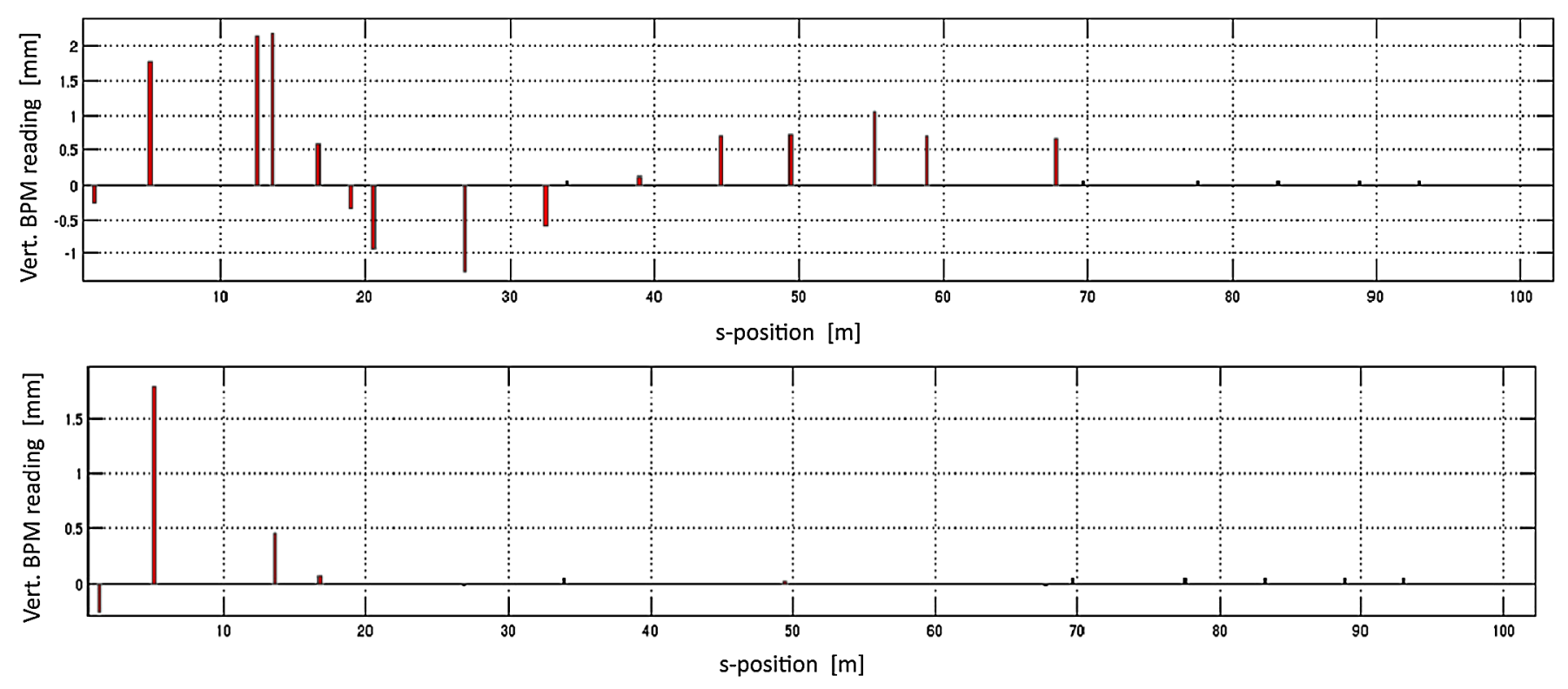

FIG. 9. Vertical trajectory in the FERMI linac, from the LH to the BC1 area, as measured and displayed by the control system. Each bar shows a BPM reading. The top plot is before correction. The bottom plot is after correction (most of BPMs reading have been forced to zero) carried out with the theoretical TRM shown in Fig. 8. The three BPMs with large bars still after correction (left side of the plot) have been excluded from the steering algorithm.

with another is avoided by verifying the orthogonality of the TRMs. This is done by merging the matrices into a global one, representing it graphically and verifying that the different blocks, representing BPMs sensitivity to steering magnets excitation, are diagonal. We point out that, in general, if the loops do not have any overlap in terms of BPMs in their response matrices, they simply do not care what the other loops (downstream) may see. So, an upstream loop will affect the downstream one. But, if the last two BPMs of an upstream loop do not have a corrector
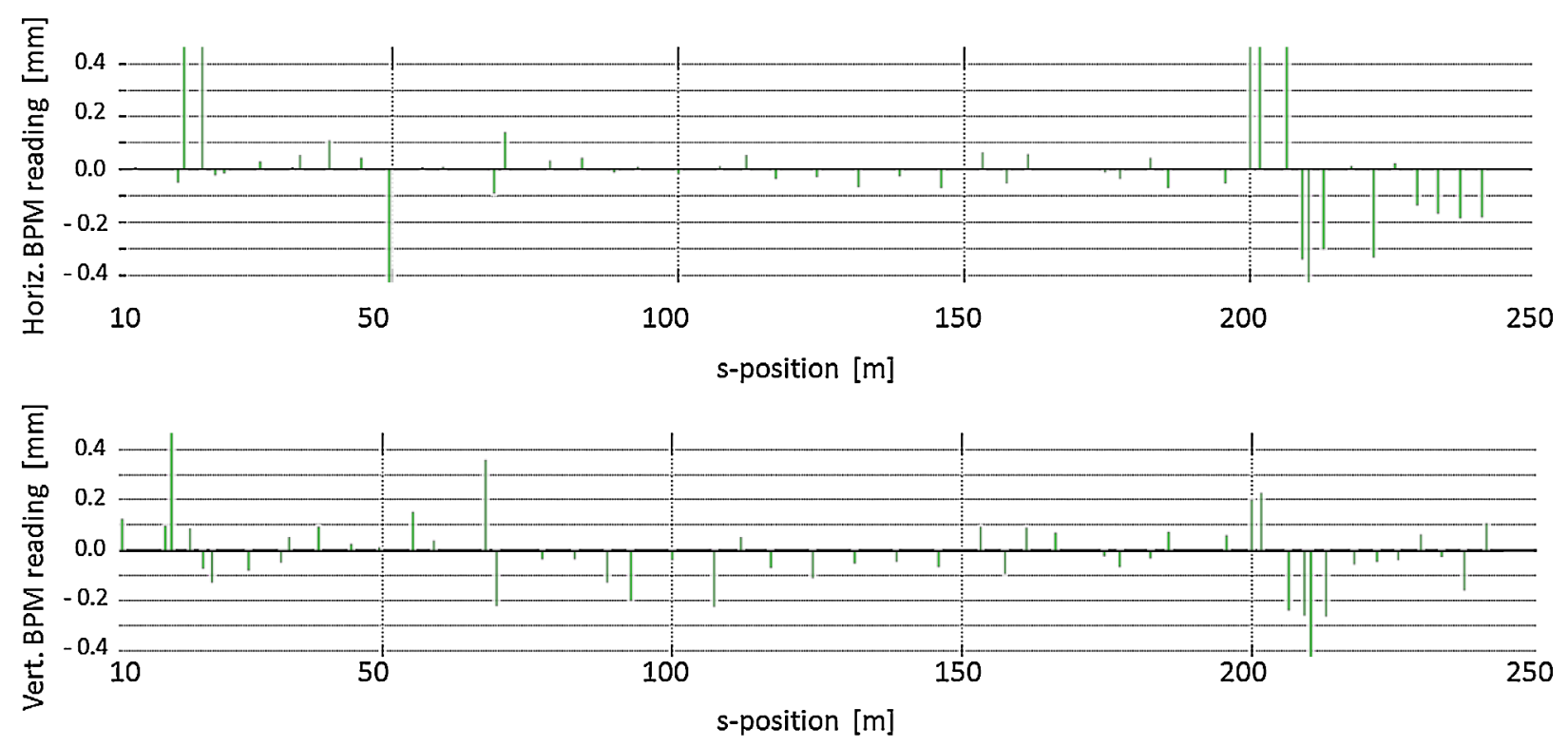

FIG. 10. Trajectory correction in the horizontal (top) and vertical plane (bottom), from the injector end to the linac end, as measured and displayed by the control system. The very large bars in the $\mathrm{LH}$ area ( $s$ position $\sim 20 \mathrm{~m})$ and $\mathrm{BC} 1$ area $(s$ position $\sim 50 \mathrm{~m}$ ) correspond to bad BPMs reading; these are excluded from the steering algorithm. The large bars in the right side of the plot $(s$ position $>200 \mathrm{~m}$ ) are in the transfer line area that was not included in the steering algorithm at the time of this measurement. The correction has been performed with the theoretical TRM shown in Fig. 8. 
between them, both position and angle will be held steady and any impact on downstream loops will be minimal. This is the procedure adopted in FERMI at the end of the linac and of the high energy transfer line, so that the linac, the transfer, and the undulator lines are insulated. Up to three feedback loops run continuously, in both planes, for a few hours: the first loop works with a $N \times M$ dimensional TRM that is a merge of the experimental matrix of the injector ( $N=M=2$, in each plane) and of the ELEGANT-based, theoretical matrix of the rest of the linac $(N=M=27$, in each plane). The second loop has been working with the ELEGANT-based, theoretical TRM of the high energy transfer line ( $N=M=11$, in each plane). The third loop has been working with an experimental TRM of the FEL1 undulator line ( $N=M=7$, in each plane).

We stress that an efficient trajectory correction with a relatively large theoretical TRMs, defined over most of the $300 \mathrm{~m}$ long lattice, is an indication of good agreement between the theoretical optics (quadrupole strengths) and the real configuration of the magnetic lattice. In particular, some modeling details such as the edge focusing of the $\mathrm{BC} 1$ dipole magnets and the rf edge focusing are also indirectly validated. The importance of this result relies on the fact that a high accuracy in the machine modeling is vital for basic optics verification and, more in general, control of the particle dynamics. A more direct proof of the agreement of the ELEGANT model with the real machine is given, as an example, in Fig. 8. It compares the measured and theoretical TRMs for the whole beam line, in both transverse planes. The experimental and the theoretical matrices show the same topology (same "peaks" and "valleys" for the same areas of sensors and actuators). During the FERMI commissioning, the theoretical matrix has successfully been used for trajectory correction. An example is given in Figs. 9 and 10.

\section{CONCLUSIONS}

An ELEGANT-based on-line control of the beam optics and of the trajectory in the FERMI electron beam delivery system has been implemented during the commissioning of the FERMI FEL. This control relies on the machine model implemented in the ELEGANT code. ELEGANT is interfaced with the real quadrupole magnets through SDDSto-MATLAB instructions and Tango server command lines. ELEGANT is used to perform optics matching, to transport the Twiss parameters along the line and for off-line comparison of different optics solutions. The success of the optics matching loop has been verified in two independent ways: first, by measuring the betatron mismatch parameter (in many cases, the error on the mismatch parameter is as small as $10^{-3}$ ); second, by measuring the beam energy spread, at different machine locations, as a function of the optics set up. In this case, the successful implementation of the optics matching has allowed sub-keV accuracy for the measurement of the energy spread. ELEGANT has also been used for the production of theoretical trajectory response matrices. The theoretical matrices have been uploaded into the trajectory feedback tool and used for local and global trajectory correction, even with parallel correction loops. A high accuracy in modeling the accelerator has thus shown itself to be important not only for basic optics verification, but also for beam trajectory control and, by extension, control of the final beam quality.

\section{ACKNOWLEDGMENTS}

The authors are in debt with G. Gaio and A. Lutman who participated in the development of the trajectory feedback tool. One of the authors (S. Di Mitri) thanks L. Giannessi for a stimulating discussion on the energy spread measurement accuracy. The authors thank the participants to the FERMI commissioning for having tested the tools for optics and trajectory control described in this article and for having given so many important suggestions for their improvement. In particular, we acknowledge: G. Penco, who is the main author of the MATLAB tools for emittance and energy measurement; S. Ferry and S. Krecic, who are the authors of the MATLAB tool for trajectory visualization; M. Trovo', who has realized the database to propagate the mean energy information through the electron beam line. Such tools have extensively been used to measure the beam Twiss parameters during optics matching, the beam energy spread in the spectrometer lines, to visualize the beam trajectory, and to properly set the energy parameter in the Tango devices.

\section{APPENDIX: ENERGY SPREAD MEASUREMENT ACCURACY}

We present here an exact treatment of the error of the energy spread measurement. We demonstrate how sub-keV accuracy has been reached for a properly matched beam. Starting from Eq. (3), we compute the relative energy spread in the exact form:

$$
\sigma_{\delta}=\frac{\sqrt{\sigma^{2}-\varepsilon \beta}}{\eta},
$$

where all optical functions refer to the bending plane. The maximum absolute error of $\sigma_{\delta}, \Delta \sigma_{\delta}$, is given by

$$
\begin{aligned}
\Delta \sigma_{\delta}= & \left|\frac{\partial \sigma_{\delta}}{\partial \sigma}\right| \Delta \sigma+\left|\frac{\partial \sigma_{\delta}}{\partial \varepsilon}\right| \Delta \varepsilon+\left|\frac{\partial \sigma_{\delta}}{\partial \beta}\right| \Delta \beta \\
& +\left|\frac{\partial \sigma_{\delta}}{\partial \eta}\right| \Delta \eta
\end{aligned}
$$

where

$$
\begin{array}{ll}
\left|\frac{\partial \sigma_{\delta}}{\partial \sigma}\right|=\frac{\sigma}{\eta^{2} \sigma_{\delta}} ; & \left|\frac{\partial \sigma_{\delta}}{\partial \varepsilon}\right|=\frac{\beta}{2 \eta^{2} \sigma_{\delta}}
\end{array}
$$


We now assume uncorrelated errors:

$$
\Delta \sigma_{\delta}=\sqrt{\left(\frac{\sigma^{2}}{\eta^{2} \sigma_{\delta}}\right)^{2}\left(\frac{\Delta \sigma}{\sigma}\right)^{2}+\left(\frac{\beta \varepsilon}{2 \eta^{2} \sigma_{\delta}}\right)^{2}\left(\frac{\Delta \varepsilon}{\varepsilon}\right)^{2}+\left(\frac{\beta \varepsilon}{2 \eta^{2} \sigma_{\delta}}\right)^{2}\left(\frac{\Delta \beta}{\beta}\right)^{2}+\sigma_{\delta}^{2}\left(\frac{\Delta \eta}{\eta}\right)^{2}} .
$$

Given the absolute energy spread $\sigma_{E}=E \sigma_{\delta}$, where $E$ is the beam mean energy, the error on its central value is

$$
\Delta \sigma_{E}=\sqrt{\sigma_{\delta}^{2} \Delta E^{2}+E^{2} \Delta \sigma_{\delta}^{2}}
$$

The absolute error on the mean energy measurement, $\Delta E$, is dominated by the screen system mechanical alignment, $\Delta=300 \mu \mathrm{m}$. Thus, we have

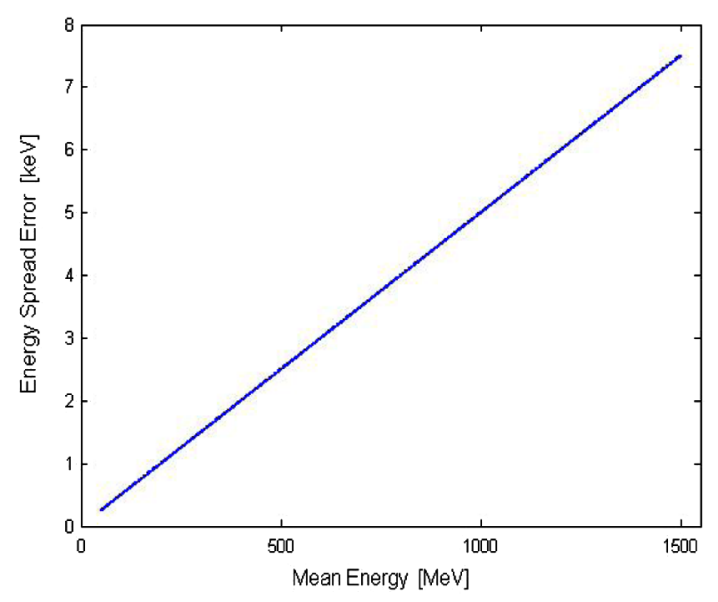

$$
\Delta E \cong E \frac{\Delta}{\eta}
$$

We now assume an optically matched beam and we evaluate the error (A5) of the absolute energy spread measurement. For typical values at the screen such as $\gamma \varepsilon=1 \mathrm{mmmrad}, \quad \beta=1 \mathrm{~m}, \quad \eta=1 \mathrm{~m}, \quad$ and $\sigma_{\delta}=$ $0.1 \%-1 \%$, the beam size is dominated by the chromatic contribution and ranges $\sigma=1-10 \mathrm{~mm}$. The error on

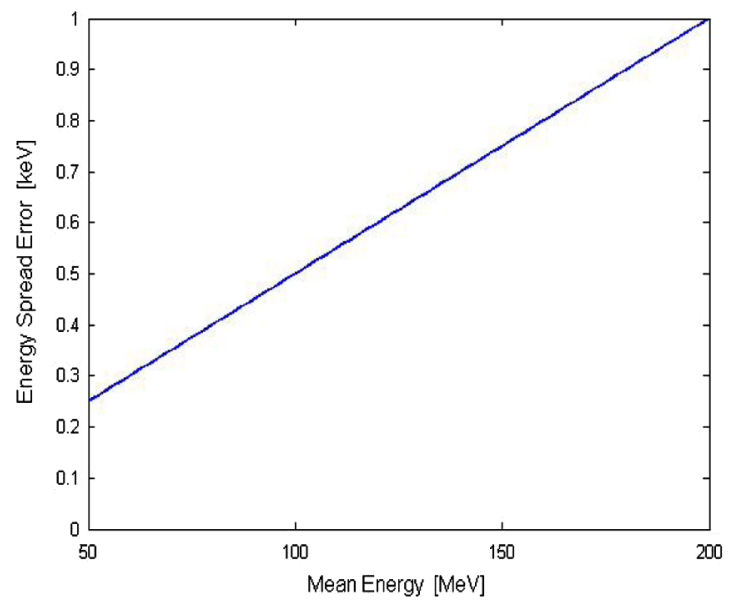

FIG. 11. Error of the energy spread measurement as function of the mean energy. A resolution of $30 \mu \mathrm{m}$ of the diagnostic device is assumed. Right plot, zoom on the low energy region.
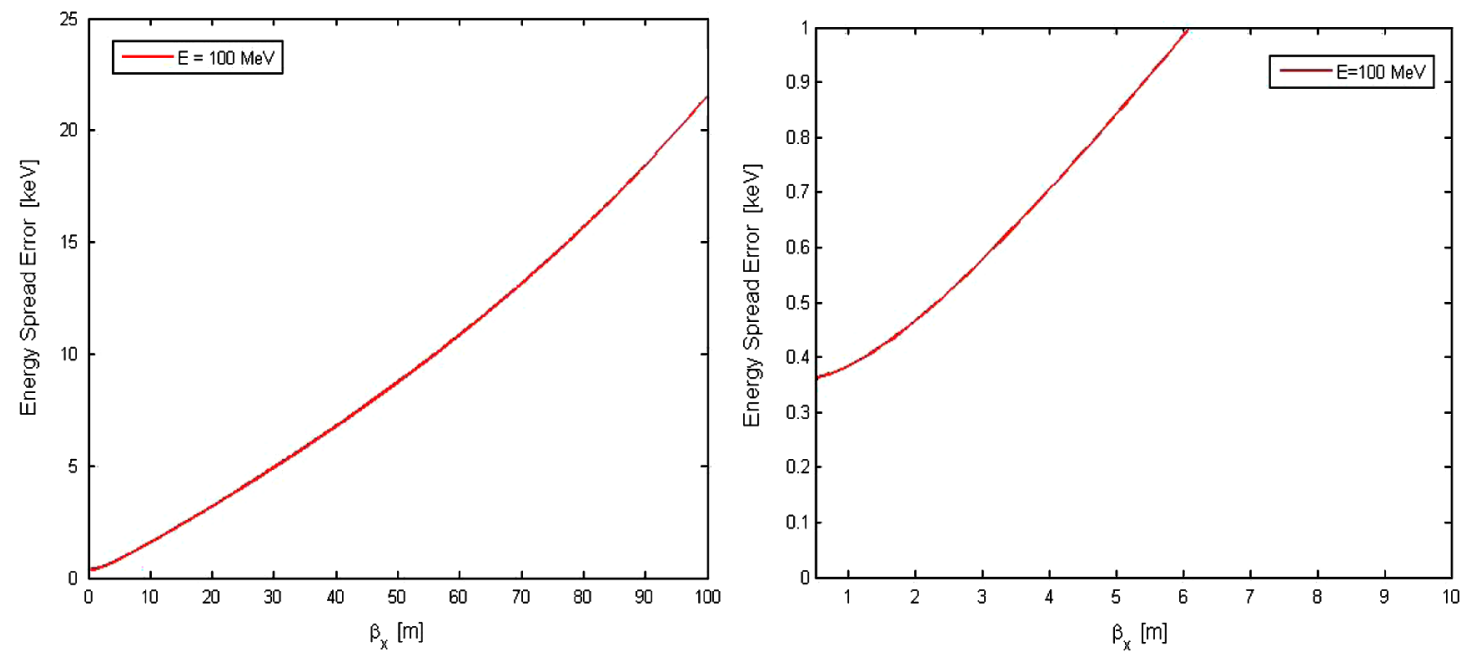

FIG. 12. Error on the energy spread measurement as function of the betatron function at the screen. A mean energy of $100 \mathrm{MeV}$ is considered. Right plot, zoom on the low beta region. 
the beam size measurement, $\Delta \sigma$, is dominated by the resolution of the screen system that is $30 \mu \mathrm{m}$. Assuming that the full spot size on the screen includes at least $6 \sigma$, we have $\Delta \sigma=5 \mu \mathrm{m}$. For the error on the geometric emittance we keep $\Delta \varepsilon=0.05 / \gamma \mathrm{mm} \mathrm{mrad}$ as it results from the quadrupole scan. In the same way, for the error on the betatron function we keep $\Delta \beta=0.01 \mathrm{~m}$. The error on the dispersion function can be estimated by considering that $\eta \sim \theta L$, where $\theta$ is the bending angle and $L$ is the distance of the observation point (screen) from the spectrometer. So, for any given geometry of the line, $\Delta \eta / \eta \approx \Delta \theta / \theta \approx$ $\Delta B / B \approx 10^{-4}$.

Inserting these values into (A4) we obtain that: the two terms proportional to $\beta$ are 2 orders of magnitude smaller than the beam size term proportional to $\sigma^{2}$; the term proportional to $\Delta \eta$ is 1 order of magnitude smaller than that. So, (A4) is effectively dominated by the beam size relative error (this is in turn determined by the resolution of the screen system) times the relative energy spread:

$$
\Delta \sigma_{\delta, \text { matched }} \cong \sigma_{\delta} \frac{\Delta \sigma}{\sigma}
$$

By substituting (A7) in (A5) and considering that $\sigma_{\delta} \Delta E$ is negligible, we find for a matched beam

$$
\Delta \sigma_{E \text {, matched }} \cong E \Delta \sigma_{\delta \text {, matched }} \cong \sigma_{E} \frac{\Delta \sigma}{\sigma} \equiv E \frac{\Delta \sigma}{\eta}
$$

Figure 11 plots Eq. (A8) for the energy range $E=50-1500 \mathrm{MeV}$. It shows that sub-keV energy spread accuracy can be reached for $E \leq 200 \mathrm{MeV}$. Figure 12 depicts the degradation of the accuracy of the energy spread measurement when the beam is mismatched. In this case we consider $\beta \approx \Delta \beta \approx 50 \mathrm{~m}$. The sub-keV accuracy, which is reached in the SPLH line at $100 \mathrm{MeV}$, is no more possible with such mismatched optics.

[1] C.J. Bocchetta et al., Report No. ST/F-TN-07/12, Sincrotrone Trieste, 2007 [http://www.elettra.trieste.it/ FERMI/index.php?n=Main.CDRdocument].

[2] L. H. Yu et al., Nucl. Instrum. Methods Phys. Res., Sect. A 393, 96 (1997).

[3] L. H. Yu et al., Science 289, 932 (2000).

[4] L. H. Yu et al., Phys. Rev. Lett. 91, 074801 (2003).

[5] G. De Ninno et al., Phys. Rev. Lett. 101, 053902 (2008).

[6] M. Borland, Report No. APS LS-287, 2000.

[7] M. Borland, in Proceedings of the 1997 Particle Accelerator Conference, Vancouver, BC (IEEE, New York, 1998), 6P020.
[8] H. Shang et al., in Proceedings of the 20th Particle Accelerator Conference, Portland, OR, 2003 (IEEE, New York, 2003), FPAG004.

[9] L. Emery, M. Borland, and H. Shang, in Proceedings of the 20th Particle Accelerator Conference, Portland, OR, 2003 (Ref. [8]).

[10] R. Soliday, S. Pasky, and M. Borland, in Proceedings of the 20th International Linac Conference, Monterey, CA, 2000 (SLAC, Menlo Park, CA, 2000), TUC14.

[11] See, for example, A. Gotz et al., MP705, ICALEPCS 2003, Gyeongiu, Korea (2003) and more information in http://www.tango-controls.org/.

[12] M. Sands, Report No. SLAC-AP-85, 1991.

[13] M. Huning and H. Schlarb, in Proceedings of the 20th Particle Accelerator Conference, Portland, OR, 2003 (Ref. [8]), WPAB017.

[14] P. Piot et al., in Proceedings of the European Particle Accelerator Conference, Vienna, 2000 (EPS, Geneva, 2000), MOP4B14.

[15] C. Beherens and Ch. Gerth, in Proceedings of the 32nd International Free Electron Laser Conference (Malmo, Sweden, 2010), MOPC08.

[16] Z. Huang, K. Bane, Y. Ding, and P. Emma, Phys. Rev. ST Accel. Beams 13, 092801 (2010).

[17] J. Safranek, Nucl. Instrum. Methods Phys. Res., Sect. A 388, 27 (1997).

[18] F. Brinker et al., in Proceedings of the 9th European Particle Accelerator Conference, Lucerne, 2004 (EPSAG, Lucerne, 2004), TUPLT035.

[19] Y. Funakoshi et al., Phys. Rev. ST Accel. Beams 10, 101001 (2007).

[20] J. Wu et al., in Proceedings of the 23rd Particle Accelerator Conference, Vancouver, Canada, 2009 (IEEE, Piscataway, NJ, 2009), WE5RFP046.

[21] S. Sasaki, K. Miyata, and T. Takada, J. Appl. Phys. 31, L1794 (1992).

[22] S. Di Mitri et al., Nucl. Instrum. Methods Phys. Res., Sect. A 608, 19 (2009).

[23] G. Penco et al., in Proceedings of the 11th European Particle Accelerator Conference, Genoa, 2008 (EPS-AG, Genoa, Italy, 2008), TUPC077.

[24] S. Di Mitri et al., in Proceedings of the 32nd International Free Electron Laser Conference (Malmo, Sweden, 2010), MOPA02.

[25] G. Penco, in Proceedings of the IPAC'10 Conference, Kyoto, Japan (ICR, Kyoto, 2010), TUOARA02.

[26] E. M. Allaria, P. Craievich, S. Di Mitri, G. Penco, and M. Trovo', in Proceedings of the SPIE Optics + Optoelectronics Conference 8078: Advances in X-ray Free-Electron Lasers (SPIE, Prague, CZ, 2011).

[27] P. Craievich et al., in Proceedings of the 32nd International Free Electron Laser Conference (Malmo, Sweden, 2010), THPC11.

[28] See http://www.leica-geosystems.com/en/Laser-TrackerSystems_69045.htm. The model that has been used for FERMI is LTD600.

[29] C. Scafuri, in Proceedings of the 12th International Conference on Accelerator and Large Experimental Physics Control System (Kobe, Japan, 2009), TUP037. 
[30] A. W. Chao and M. Tigner, Handbook of Accelerator Physics and Engineering (World Scientific, Singapore, 2006), 3rd ed.

[31] M. G. Minty and F. Zimmermann, Report No. SLAC-R621, 2003.

[32] G. Penco et al., in Proceedings of the 11th European Particle Accelerator Conference, Genoa, 2008 (Ref. [23]), TUPC079.

[33] http://www.mathworks.com/products/matlab/.

[34] R. Akre et al., Phys. Rev. ST Accel. Beams 11, 030703 (2008).
[35] P. Craievich, S. Di Mitri, and A. A. Zholents, Nucl. Instrum. Methods Phys. Res., Sect. A 604, 457 (2009), and references therein.

[36] L. N. Trefethen and D. Bau, Numerical Linear Algebra (Society for Industrial and Applied Mathematics, Philadelphia, 1997).

[37] P. C. Hansen, BIT 27, 534 (1987).

[38] A.N. Tychonoff and V.Y. Arsenin, Solution of Ill-posed Problems (Winston \& Sons, Washington, 1977). 